\title{
Interviews with Upper-Level Undergraduates about Electromagnetic Plane Waves
}

\author{
Andrew J. Berger \\ The Institute of Optics, University of Rochester, Rochester, NY 14627
}

\begin{abstract}
A robust grasp of electromagnetic (EM) plane waves is helpful for studying advanced Optics topics such as reflection, interference, and the wavelength dependence of refractive index. Although there have been many studies of students' understanding of waves, only a small fraction have been dedicated to plane waves, which are particularly challenging both conceptually and mathematically. In this study, nine upper-level science/engineering majors were prompted to explain how a provided mathematical representation of an EM plane wave dictated something conceptually planar. An unexpected initial finding is that students' initial responses always went outside the realm of the prompt: they either focused on aspects of the mathematics that were unrelated to planes, or they relied upon outside information to make the connection to planes. This revealed difficulty in blending the conceptual and mathematical representations.
\end{abstract}

PACS: 01.40.Fk, 01.40.Gb, 01.42, 41.20.Jb

\section{INTRODUCTION}

Electric field plane waves are a core concept in the teaching of Optics. Various properties of plane waves are invoked in teaching elementary topics such as interference fringes and Fresnel reflection. At the more advanced level, monochromatic plane waves provide a mathematical basis set for discussions of diffraction, spatial frequencies in images, optical Fourier transformation, and more complicated waves such as pulses.

Because electric field plane waves assign time-varying vectors to all points in space, conceptual depictions of them can be only imperfectly rendered on blackboards or even on animated screens [1]. The standard mathematical representation of plane waves using complex exponentials is also troublesome for typical undergraduate students, even at the junior level. We hypothesize that if students build stronger connections between the conceptual and mathematical representations, their facility with plane waves will increase. More generally, Kuo et al. [2] and Hull et al. [3] have advanced the notion that "blended" conceptual and formal mathematical reasoning is an important dimension of physics expertise.

A technique for strengthening students' reasoning skills is to confront them with multiple representations of a concept and challenge them to reconcile the different representations. Linenberger and Bretz [4] exploited this approach in studying student understanding of enzyme/substrate interaction, using what they called a 3-phase single-interview technique with multiple representations (3PSIT-MR). In the first phase, students were asked to describe their initial understanding of enzyme/substrate interaction; in the second, they were given a particular representation (sketch) and asked to describe its salient points, and then repeated these steps when shown a second, related visual representation. In the third phase, the students were forced to reconcile cognitive dissonance caused by the different features of the two models, either by rejecting parts of one model or by adjusting their own conceptual model.
This study used the 3PSIT-MR format to explore how students reconcile conceptual and mathematical representations of an electromagnetic plane wave. Ambrose et al. [1] used essentially this approach in interviewing students about the direction of electromagnetic plane wave propagation, allowing them to start either with the cross product $\vec{E} \times \vec{B}$ or with the wave implications of the mathematical form $(k x+v t)$ but then requiring them to reconcile this with the other approach. This work follows in the same vein but focuses particularly upon the planar nature of the wavefronts.

\section{STUDY DESIGN}

This study consisted of one-on-one interviews with nine Optics or Physics majors (seven seniors and two earlystage graduate students), each of whom had previously taken an upper-level undergraduate course in electromagnetic theory in which electric field plane waves were discussed. The series of prompts for the semi-structured interview was as follows:

1. Oral only (no visuals): How would you explain electromagnetic plane waves to a fictional Optics student at the sophomore or junior level?

2. (Revealing the cartoon in Figure 1) How would you use this conceptual representation to teach this student about electromagnetic (EM) plane waves?

3. (Concealing the cartoon and revealing the mathematical expression in Figure 2) How would you use this mathematical representation to teach the student about EM plane waves?

4. (Revealing both pictures) How do these two representations work together to teach about EM plane waves?

5. What possible misunderstandings about plane waves might be caused by these pictures?

The interview subjects were repeatedly prompted to teach another (invisible) student in order to reduce their feeling of being tested and enable them to speak more 
candidly. The prompts were intentionally open-ended because the students' choices of starting points were of primary interest. Subjects all expressed familiarity with the meaning of variables chosen in the mathematical expression; none asked for further clarification or discussed them in incorrect ways.

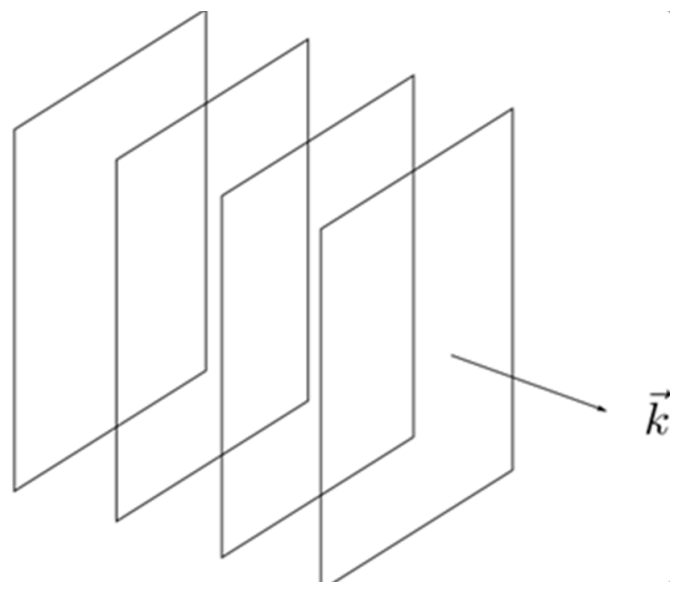

FIG. 1. Conceptual representation of a plane wave as a stack of squares in three-dimensional space with an associated wavevector.

$$
\overrightarrow{E_{0}} e^{i(\vec{k} \cdot \vec{r}-\omega t)}
$$

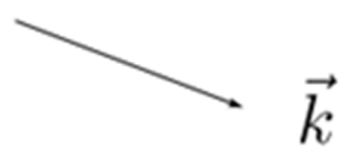

FIG. 2. Mathematical representation of a plane wave as a complex exponential multiplied by a constant vector $\vec{E}_{0}$.

Interviews 3 through 9 also included some form of the following question: "What in the math expression implies planes?" This question emerged naturally in the first few interviews and became standard after that, once students' difficulty with the question became apparent.

Interview subjects were seated at a table with a large square whiteboard surface available to write upon with multiple markers. The large writing on the whiteboard, as opposed to pencil and paper, made it easier to capture video of the writing via a video recorder placed on the top of a bookshelf. The recorder also obtained audio, which was subsequently transcribed. The .MPEG file and transcript were imported into a software program (NVivo) for analysis. Each interview lasted 30-45 minutes.

The transcripts are being analyzed using an emergent coding approach. This is still in progress.

\section{RESULTS}

\section{A. Not answering "Where are the planes?"}

When students 3-9 were asked "How does the math expression dictate planes?", their initial responses tended not only to be wrong, but not to stay on the topic of the question. Four types of insufficient answers were proposed by more than one student:

1. "The dot product term dictates a sinusoidally varying phase in the direction parallel to the wavevector." (not addressing why there are planes) [students $4,7,8]$

2. "The amplitude $E_{0}$ is constant, therefore there are planes." (incorrect and not justified) $[4,8]$

3. Student highlights the dot product or sinusoidal variation, but cannot say why it implies planes. $[3,6,9]$

4. Student invokes different types of planes, e.g. the real-imaginary plane (not relevant), the plane defined by orthogonal $\mathrm{E}$ and $\mathrm{B}$ fields (not contained in the math), the plane of possible linear polarizations (not contained in the math). [5,8,9]

Most of these answers stray from the request to connect the mathematical expression to the relevance of planes. A sophisticated response to this request might be, "How does this expression change as I vary position $\vec{r}$, and would the surfaces of equal value be planar?" Varying $\vec{r}$ in the direction of $\vec{k}$ would quickly be seen as unfruitful. The many students who offered answer \#1, however, pursued this path without checking whether it was addressing the question. Conversely, students using answer \#4 stayed focused on the issue of planes but did not confine themselves to the particular math expression under discussion, as an expert likely would. Instead, they invoked either generalities about imaginary exponentials or outside information about magnetic fields.

Later in their interviews, three of the students eventually arrived at robust answers. All such answers included (a) an awareness that all locations $\vec{r}$ in a plane parallel to the wavevector give the same value of the dot product $\vec{k} \cdot \vec{r}$, and (b) the statement that the points in a given plane would have the same phase. It is not known how many other students might have also reached this point, as arriving at it was not a goal of the interview protocol.

\section{B. Illustrative student remarks}

Several students made clear-sounding statements about the planes being surfaces of equal phase, but then failed to access this information when asked how the mathematics implied planes. 


\section{Student A: contradictory statements}

Prior to receiving any visual prompt, Student A offered a correct-sounding statement about planar surfaces of constant phase:

So you could, say, sketch at some certain time $t_{0}$ and it would, you know, look something like a sine wave. I guess that would be the real part or whatever, and you could define, say, one of these points, I think they sometimes call it a phase front, and you could say if you'd imagine this way of propagating in space, the reason we call it a plane wave is that point of constant phase is a plane, so you get these, like, a little plane's going like this [hands advancing planes through the air].

Figure 3 shows what the student had sketched at this juncture. The tick mark on the sine wave is the "point" to which the student refers (planes were frequently associated with points, as if a plane normal to the $z$-axis intersected the sine wave at that point).

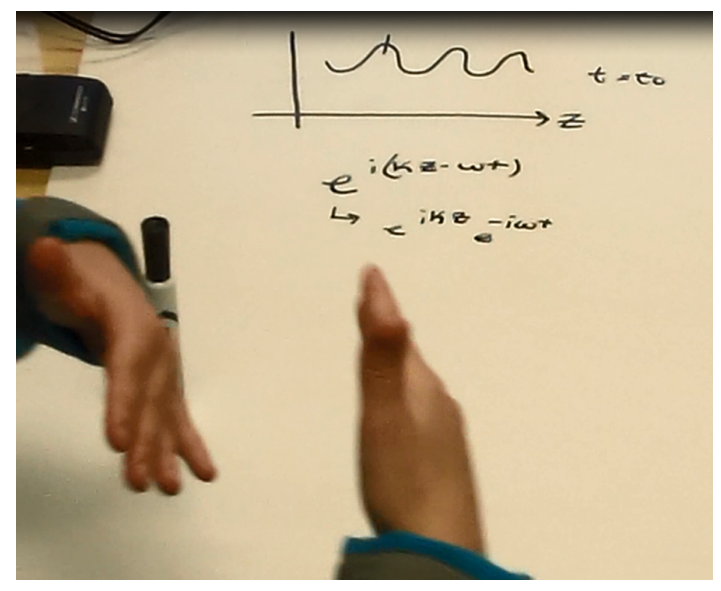

FIG. 3. Sketches made by Student A during initial phase of interview. Hands are indicating planes marching in the $+z$ direction.

During the latter part of the interview, however, after being asked to make connections between the conceptual and mathematical representations of plane waves, Student A said:

So the planes are, sort of, I guess you could say, are artificially constructed. It's not like the planes are a fundamental property of this wave.... We only get those planes because we arbitrarily choose this, like, surface of constant phase and then we track where it goes, and that's where you get the planes.

The student says we focus on planes only as a convention, and thus feels no obligation to seek something specific to planes in the mathematical expression. As shown in Figure 4, by this point in the interview Student A had sketched many sine waves, emphasizing the axial variation of the plane wave's phase but not drawing any example of planes (insufficient answer type \#1 from above).

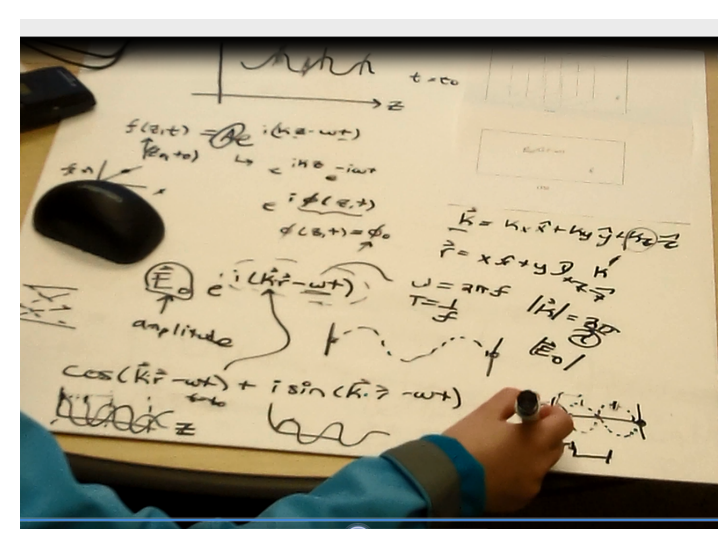

FIG. 4. Student A sketched many sine waves and mathematical expressions, but never diagrammed the planar aspect of electric field plane waves.

\section{Student B: alternative planes}

Student B, when asked how the mathematics implied planes, focused attention on the various orientations that the electric field could have at a single point. B discussed the vertical line swept out by the linear polarization over time, and that there could be different polarizations, all perpendicular to the wavevector. This set of possible lines, associated with a single point in the field, then defines a square like the one seen in the conceptualrepresentation prompt:

Interviewer: I don't get why this [the equation] would be a plane wave. I hear about either cosines and electric fields, but I don't see why there's a plane. What could we say to this student about why there are planes here?

Student B: ...So you have a direction $\vec{k}$, and your $\vec{E}$ is perpendicular to your $\vec{k}$, and so if your $\vec{E}$ is oscillating, so, it's traveling this direction and it's oscillating, and your $\vec{E}$ vector points this way, but it kind of goes all the way out, so....

I: Sometimes it's higher and sometimes it's lower, okay

S: Right, and so your plane, you have your plane because $\vec{E}$ is filling all space around $\vec{k} \ldots . \vec{E}$ can be perpendicular all around, I guess. 
I: What does that mean, "perpendicular all around"?

S: Like 'cause $\vec{E}$ is perpendicular to $\vec{k}$ so, like, it's gonna be this way, right, or another direction, I guess [indicating various linear polarization options]

I: All right, so it could be pointing anywhere in a plane

S: Yeah, I don't know, maybe that's why people like the squares [from the first visual prompt].

The associated picture drawn by the student is shown in Figure 5. Student B's answer is type \#4: although it does refer to planes, it derives planes using outside information (electric field being allowed in any orientation normal to the wavevector). While the orientation of the plane is drawn correctly, the student has incorrectly identified a plane in electric field space (the plane of possible field polarizations) rather than a plane in Cartesian space (the locus of points with the same phase).

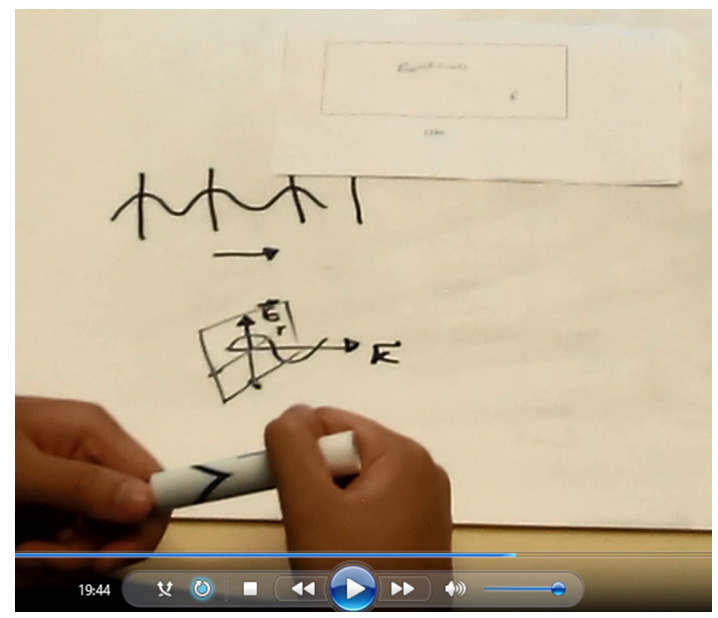

FIG. 5. Student B sketched a plane composed of a box that "holds" multiple possible linear polarizations, thus defining a plane. Note that this student employed the same sine-wave picture to depict the plane wave as used by Student A.

\section{CONCLUSIONS}

No student offered an initially correct answer to the question of how the mathematical expression for an electric field plane wave dictates planes. In most types of incorrect response, students ignored a key aspect of the question itself, either the precise form of the mathematical expression or the geometrical nature of the planes. In one sense, one can regard this as behavior that resolves cognitive dissonance, which is what the 3PSIT-MR interview is intended to explore. In several instances, including the excerpts shown from Student A's interview, a correct conceptual description of plane waves having planar iso-phase surfaces was abandoned once a mathematical expression was placed in view.

The tendency of the students to ignore the "rules of the question" merits further investigation. It could be that this behavior is indicative of a novice/expert disparity. As noted earlier, Kuo et al. [2] and Hull et al. [3] suggest that "blended" conceptual and formal mathematical reasoning is a hallmark of physics expertise. Here, the students' willingness to stop short of answering the question suggests some difficulty in blending their conceptual and formal mathematical reasoning. Perhaps, however, the students themselves recognized that their answers were incomplete; future interviews could investigate this.

Future research could also investigate whether students answer more correctly (without additional prompting) if the question is preceded by an exercise in relating mathematical expressions to conceptual diagrams. Further analysis of students' specific mental images of plane waves, and the difficulty of linking these to the mathematical expression, will also be performed.

\section{ACKNOWLEDGEMENTS}

This work is supported in part by an NSF Research Initiation Grant for Engineering Education, award EEC1240277. The author thanks Professors Scott Franklin and Ben Zwickl (Rochester Insititute of Technology) for mentoring and the RIT SMERC (Science, Math, and Engineering Research Collaborative) for fruitful discussions.
${ }^{1}$ B. Ambrose, P. Heron, S. Vokos, and L. McDermott, "Student understanding of light as an electromagnetic wave: Relating the formalism to physical phenomena," Am. J. Phys. 67(10), 891-898 (1999).

${ }^{2}$ E. Kuo, M. M. Hull, A. Gupta, and A. Elby, "How students blend conceptual and formal mathematical reasoning in solving physics problems," Science Education 97(1), 3257 (2013).
${ }^{3}$ M. M. Hull, E. Kuo, A. Gupta, and A. Elby, "Problemsolving rubrics revisited: Attending to the blending of informal conceptual and formal mathematical reasoning," Phys. Rev. ST Phys. Educ. Res. 9, 010,105 (2013).

${ }^{4}$ K. J. Linenberger and S. L. Bretz, "Generating cognitive dissonance in student interviews through multiple representations," Chem. Educ. Res. Pract. 13, 172-178 (2012). 